\title{
Pessaries and rectovaginal fistulae: consequences of delayed clinical follow-up in the Covid-19 pandemic
}

\author{
Jordan Mendelson $^{1} \cdot$ Bogdan Grigorescu ${ }^{1,2}$ - Catherine Quinn ${ }^{2} \cdot$ George Lazarou $^{1,2}$
}

Received: 25 May 2021 / Accepted: 16 June 2021 / Published online: 8 July 2021

(C) The International Urogynecological Association 2021

\begin{abstract}
Introduction and hypothesis While 2017 guidelines from The American College of Obstetricians \& Gynecologists called for pessary replacement every 3 to 4 months, a recent study in Obstetrics and Gynecology suggested that uninterrupted pessary use up to 6 months is not an independent risk factor for development of pessary-related complications.

Methods Our recent experience throughout the Covid-19 pandemic highlights the potential ramifications of delayed clinical follow-up.

Results During the Covid-19 pandemic, 3 of our patients developed rectovaginal fistulae secondary to Gellhorn pessary erosion in the context of delayed clinical follow-up. Our patients had previously attended routine appointments every 3 months without complications until missed appointments secondary to the pandemic led to fistulae formation.

Conclusion We believe that delayed clinical follow-up of pessary management beyond 3 months due to the Covid-19 pandemic may lead to fistula complications in elderly women with Gellhorn pessaries.
\end{abstract}

Keywords Complication $\cdot$ Covid-19 $\cdot$ Delay $\cdot$ Fistula $\cdot$ Gellhorn $\cdot$ Pessary

\section{Introduction}

Vaginal pessaries are considered to be an appropriate conservative and minimally invasive option for symptomatic treatment of pelvic organ prolapse and/or urinary incontinence in women [1]. Pessaries have been shown to reduce symptoms of urinary incontinence, vaginal bulge, and pelvic pressure in women by $22-46 \%, 70-90 \%$, and $29-49 \%$, respectively [2-4]. We have previously reported on the benefits of pessary use in patients with advanced pelvic organ prolapse [5]. Pessaries are primarily made of medical-grade silicone and come in various shapes and sizes; these include ring, Gellhorn, cube, Shaatz, Inflatoball, and donut pessaries, among others [4].

Current literature varies dramatically in its estimation of the prevalence of complications associated with pessaries. While

George Lazarou

George.Lazarou@nyulangone.org

1 New York University Long Island School of Medicine, Mineola, NY, USA

2 Department of Obstetrics and Gynecology, New York University Langone Hospital-Long Island, Mineola, NY, USA one study found that $73.1 \%$ of women using a pessary experienced at least 1 complication, another found that $88.5 \%$ of women experienced no complications [6, 7]. Some of the more commonly cited complications include vaginal erosions and vaginal infection $(8.9 \%$ and $2.5 \%$, respectively, in one study) [7]. Vaginal estrogen therapy is often recommended in conjunction with a pessary in peri- and postmenopausal women to reduce the incidence of vaginal erosion [1]. Other more serious potential complications include fistula formation, fecal impaction, hydronephrosis, and urosepsis [8]. A literature review conducted in 2008 determined that $91.3 \%$ of these major complications were related to a neglected pessary [9]. As a result, 2017 guidelines from The American College of Obstetricians and Gynecologists (ACOG) called for routine clinical follow-up every 3 or 4 months for pessary replacement in women who are unable to do so independently [10]. However, a more recent study published in ACOG's Obstetrics and Gynecology determined that routine pessary follow-up every 6 months was noninferior to follow-up every 3 months based on the observed prevalence of complications of pessary use [11]. This randomized noninferiority trial included 130 women with ring, Gellhorn, or incontinence dish pessaries in place, but notably excluded women with erosion of the vaginal epithelium from participation. The study found 
that uninterrupted pessary use for up to 6 months is not an independent risk factor for development of vaginal epithelial erosion in the patient population studied. However, as the study points out, its main outcome measure was predicated on a rating system of vaginal epithelial abnormalities which has not been validated [11]. While the study claims that its results are generalizable to the majority of women undergoing office-based pessary care, this generalizability cannot extend to women with pre-existing epithelial erosion secondary to vaginal atrophy, which is likely more common in the elderly population. Regardless, this study, in conjunction with the Covid-19 pandemic, likely led to fewer routine follow-up visits for women with pessaries in place throughout the pandemic. In this case series, we highlight the potential ramifications of such a change in the guidelines for routine pessary care: within the last 9 months, 3 of our patients developed rectovaginal fistulae secondary to pessary erosion of the vaginal wall due to delayed clinical follow-up.

\section{Cases}

The first patient is a 99-year-old G2P2 female with no significant past medical history. She had her initial pessary placed prior to July 2017 for symptomatic pelvic organ prolapse with incomplete voiding. Her initial pessary was a $3 \frac{1}{2}$-inch Gellhorn long-stem pessary. From the time her pessary was placed until January 2020, she attended routine clinical follow-up appointments approximately every 3 months. Throughout that time, her pessary was changed first to a 3 1/4-inch Gellhorn and finally to a 3-inch Gellhorn because she became newly wheelchair-bound and it became increasingly difficult to remove her pessary. Beginning in November 2017 and persisting through April 2019, the patient was found to have minor vaginal spotting on removal and cleaning of the pessary at these routine follow-up appointments secondary to postmenopausal atrophic vaginitis, despite her use of vaginal estrogen therapy. At each visit, hemostasis was achieved using topical silver nitrate, and the patient was encouraged to continue using vaginal estrogen therapy to treat her atrophic vaginitis and thus prevent vaginal epithelial erosion. Throughout this period, the patient remained very pleased with her pessary and she noted normal urinary and bowel function with the pessary in place. Beginning in January 2020, the patient's routine clinical follow-ups became less frequent because of the Covid-19 pandemic. After a routine follow-up in late January, the patient returned $31 \frac{1}{2}$ months later in early May. Her physical examination was benign at this visit, and she was again encouraged to return in 3 months for further follow-up. Due to the pandemic, the patient did not return for almost $4 \frac{1}{2}$ months. In late September 2020, the patient returned to the office with complaints of vaginal discharge for 2 weeks. On physical examination, the patient was noted to have discharge of feculent material from a midline $2 \mathrm{~cm} \times 1 \mathrm{~cm}$ rectovaginal fistula secondary to pessary erosion of the posterior vaginal wall. At the time, the patient's pessary was removed without replacement and the patient was referred to colorectal surgery. Due to the patient's advanced age and increased risk of perioperative morbidity and mortality, the patient agreed to medical management of symptoms with a high-fiber diet, as-needed vaginal douching, and antibiotic prophylaxis for urinary bacterial suppression.

The second patient is an 80 -year-old G3P3 female with a past medical history of atrial fibrillation, mitral regurgitation secondary to mitral valve prolapse, recurrent urinary tract infection, pulmonary embolism, irritable bowel syndrome, Sjögren's syndrome, and Barré-Liéou syndrome. The patient initially had a $31 / 4$-inch Gellhorn long-stem pessary placed in September 2017 for symptomatic relief of cystocele, rectocele, and uterovaginal prolapse. The patient tolerated the pessary well and was satisfied with the relief of her symptoms for several years. At a routine pessary follow-up visit in March 2019, the patient was found to have an isolated episode of minor vaginal spotting on removal and cleaning of the pessary secondary to postmenopausal atrophic vaginitis. Hemostasis was achieved in the office with topical silver nitrate, and the patient was encouraged to use vaginal estrogen therapy, which she did. In February 2020, the patient developed a complicated urinary tract infection, which required inpatient antibiotic therapy. Due to the Covid-19 pandemic, this patient similarly presented for routine pessary follow-up less frequently than she had previously. The patient next presented in late June 2020 after a $41 / 2$-month hiatus for a routine pessary follow-up with an associated complaint of vaginal discharge that had persisted for 1 week, despite the use of vaginal estrogen therapy. On physical examination, the patient was found to have feculent discharge per vagina from a $1 \mathrm{~cm} \times$ $1 \mathrm{~cm}$ rectovaginal fistula secondary to pessary erosion of the posterior vaginal wall. This patient was similarly referred to colorectal surgery. The patient underwent a colonoscopy in July 2020 to rule out any inflammatory or malignant pathologic process and subsequently underwent surgical fistula repair with an advancement flap. The patient tolerated the procedure well and has experienced no fistula recurrence since. In September 2020, the patient developed stage IV uterovaginal prolapse and urinary retention secondary to anterior vaginal prolapse and was unable to use a vaginal pessary. The patient subsequently underwent a robotic-assisted supracervical hysterectomy and bilateral salpingo-oophorectomy with sacrocolpoplexy in February 2021. She tolerated the procedure well and passed an active void trial on postoperative day 1. At follow-up visits, the patient continued to do well, with no recurrence of vaginal prolapse and return of normal urinary and bowel function.

The third patient is an 86-year-old G2P2 female with a past medical history of hypertension, hyperlipidemia, 
hypothyroidism, congestive heart failure, and coronary artery disease status post placement of multiple stents. In February 2021 , the patient presented to her gynecologist with complaints of malodorous vaginal discharge. When pelvic examination revealed a 3-inch Gellhorn long-stem pessary in place, the patient stated that it had been in place for "years" to treat pelvic organ prolapse with associated urinary incontinence. The patient's healthcare proxy subsequently clarified that the patient had gone for routine pessary follow-up appointments until the beginning of the Covid-19 pandemic in March 2020, which caused the patient to miss several follow-up appointments, though she was unaware when the patient received her initial pessary. Physical examination revealed a copious amount of feculent vaginal discharge, concerning for rectovaginal fistula. The patient's pessary was removed, and she was referred to urogynecology. When she presented to our office in March, physical examination revealed a $3 \mathrm{~cm} \times 2-\mathrm{cm}$ rectovaginal fistula secondary to pessary erosion of the posterior vaginal wall. She was then referred to colorectal surgery for consultation. She currently has an appointment scheduled for late April 2021 to determine treatment options for fistula management.

\section{Discussion}

We believe that these 3 cases of pessary-induced rectovaginal fistulae illustrate the potential consequences of missed routine clinical follow-up appointments for pessary management associated with the Covid-19 pandemic, beyond the recommended 3-month interval. It is worth noting that all 3 of these patients used Gellhorn pessaries. Of the approximately 700 patients that we routinely see in our practice for pessary management, approximately 175 use Gellhorn pessaries. One study of 123 women using ring pessaries found zero incidence of major complications with uninterrupted use for 2 consecutive years [12]. A similar study found that the interval length between office-based removal and cleaning of a pessary does not impact the incidence of any complications, up to 9 months, though it only included women with ring pessaries in place. Furthermore, $45.2 \%$ of patients in the prospective cohort study performed self-management of their pessaries, likely minimizing the generalizability of the results [13]. In addition, as Melon points out in his commentary on the study, its lack of objective data is a major limitation, as the study's main outcome measure was a subjective visual analog scale [14]. Therefore, further study is warranted to examine potential differences in the incidence of fistula formation and/or other major complications among various pessary types. That Gellhorn pessaries are often used in women who require a larger pessary represents a potentially confounding variable for this future study.
Notably, all 3 of the patients presented here are of advanced elderly age, which likely contributed to some degree to their formation of rectovaginal fistulae. Likewise, all 3 of these patients had relatively large Gellhorn pessaries in place, which may present a confounding variable to our analysis, as it is possible that the size of the pessary itself represents an independent risk factor for the development of significant complications. Approximately $70 \%$ of our patients with Gellhorn pessaries in place use similarly sized Gellhorn pessaries to the 3 patients presented here, ranging from 3 to $3 \frac{1}{2}$ inches. Furthermore, while we collected information about the patients' use of vaginal estrogen therapy at each follow-up visit, it is impossible for us to accurately assess compliance within the limited patient population presented here. We routinely recommend that our pessary patients with postmenopausal atrophic vaginitis that do not have risk factors for uterine or breast cancer use $1 \mathrm{~g} 0.01 \%$ estradiol vaginal cream twice weekly at bedtime. None of our 3 patients ever complained of vaginal bleeding between follow-up appointments; rather, the superficial vaginal spotting observed at follow-up visits likely represented a direct consequence of routine pessary removal and cleaning. Similarly, none of our 3 patients were ever found to have their pessary facing knob-down into the rectum during clinical follow-up.

We have been using pessaries in our urogynecology practice for the conservative management of symptomatic pelvic organ prolapse for more than 20 years. All of our patients requiring the use of a pessary undergo a standardized fitting process. This process entails ensuring both the right type and size of pessary for each individual patient. We generally attempt to use ring pessaries as the initial pessary for most patients, especially those who are sexually active, those with anterior-predominant vaginal prolapse, and those who are willing and able to self-manage their pessaries. We use Gellhorn pessaries for patients with advanced uterus or apical vaginal prolapse and patients who had previously failed ring pessaries. Our fitting process begins with matching the diameter of the pessary with that of the vaginal genital hiatus and vaginal introitus. After initial placement of the pessary, we ensure that it sits level in the vaginal area and posterior to the symphysis pubis and that it does not protrude through the vaginal introitus either at rest or with the patient performing the Valsalva maneuver. We also ensure that there is sufficient space (generally a fingerbreadth) between the pessary edge and the vaginal wall to guarantee maximal comfort for the patient. We then assess the patient for comfort with the pessary in place in the supine, sitting, and standing positions. If these are all comfortable, the patient ambulates for $10-15$ minutes and attempts to void with the pessary in place to ensure functionality remains intact. Lastly, as a standard component of each initial pessary visit, we educate our patients about potential signs and symptoms of complication development to be cognizant of; some of these include new- 
onset vaginal pressure, pain, vaginal bleeding, and difficulty urinating. Throughout our time using pessaries, we have regularly followed our patients every 3 months for routine pessary care. None of our patients had ever developed a rectovaginal fistula secondary to pessary erosion prior to these 3 patients. Therefore, our study not only suggests that missed routine appointments associated with the Covid-19 pandemic may result in fistula complications, especially when large Gellhorn pessaries are used in the elderly population with pre-existing vaginal atrophy, but also that these serious pessary complications may be prevented by simply adhering to the recommended 3-month interval for routine pessary management. In an attempt to adhere to this recommended interval throughout the Covid-19 pandemic, we sent letters to each of our pessary patients that missed a routine pessary follow-up appointment, both urging them to continue their routine pessary care and informing them once again of signs and symptoms of concern. Moreover, this study effectively highlights another adverse health complication of interrupted routine medical care associated with the Covid-19 pandemic [15]. Further study is warranted in the form of a randomized trial to elucidate the effect of delayed routine pessary care in the elderly population, including those with pre-existing vaginal epithelium erosion, on the development of major pessary complications.

\section{Declarations}

Conflict of interest None.

\section{References}

1. Wu YM, Welk B. Revisiting current treatment options for stress urinary incontinence and pelvic organ prolapse: a contemporary literature review. Res Reports Urol. 2019;11:179-88. https://doi. org/10.2147/RRU.S191555.

2. Clemons JL, Aguilar VC, Tillinghast TA, Jackson ND, Myers DL. Patient satisfaction and changes in prolapse and urinary symptoms in women who were fitted successfully with a pessary for pelvic organ prolapse. Am J Obstet Gynecol. 2004;190(4):1025-9. https:// doi.org/10.1016/j.ajog.2003.10.711.

3. Fernando RJ, Thakar R, Sultan AH, Shah SM, Jones PW. Effect of vaginal pessaries on symptoms associated with pelvic organ prolapse. Obstet Gynecol. 2006;108(1):93-9. https://doi.org/10. 1097/01.AOG.0000222903.38684.cc.

4. Robert M, Schulz JA, Harvey MA, et al. Technical update on pessary use. J Obstet Gynaecol Can. 2013;35(7):664-74. https://doi. org/10.1016/S1701-2163(15)30888-4.

5. Powers K, Lazarou G, Wang A, et al. Pessary use in advanced pelvic organ prolapse. Int Urogynecol J. 2006;17(2):160-4. https://doi.org/10.1007/s00192-005-1311-8.

6. Bai SW, Yoon BS, Kwon JY, Shin JS, Kim SK, Park KH. Survey of the characteristics and satisfaction degree of the patients using a pessary. Int Urogynecol J. 2005;16(3):182-6. https://doi.org/10. 1007/s00192-004-1226-9.

7. Hanson LAM, Schulz JA, Flood CG, Cooley B, Tam F. Vaginal pessaries in managing women with pelvic organ prolapse and urinary incontinence: patient characteristics and factors contributing to success. Int Urogynecol J. 2006;17(2):155-9. https://doi.org/10. 1007/s00192-005-1362-x.

8. Jelovsek JE, Maher C, Barber MD. Pelvic organ prolapse. Lancet. 2007;369(9566):1027-38. https://doi.org/10.1016/S0140-6736(07) 60462-0.

9. Arias BE, Ridgeway B, Barber MD. Complications of neglected vaginal pessaries: case presentation and literature review. Int Urogynecol J Pelvic Floor Dysfunct. 2008;19(8):1173-8. https:// doi.org/10.1007/s00192-008-0574-2.

10. Practice Bulletin No. 176: Pelvic Organ Prolapse. Obstet Gynecol. 2017;129(4):e56-e72. https://doi.org/10.1097/AOG. 0000000000002016.

11. Propst K, Mellen C, O’Sullivan DM, Tulikangas PK. Timing of office-based pessary care. Obstet Gynecol. 2020;135(1):100-5. https://doi.org/10.1097/AOG.0000000000003580.

12. Miceli A, Fernández-Sánchez M, Polo-Padillo J, Dueñas-Díez JL. Is it safe and effective to maintain the vaginal pessary without removing it for 2 consecutive years? Int Urogynecol J. 2020;31(12):2521-8. https://doi.org/10.1007/s00192-020-042405.

13. Thys SD, Hakvoort RA, Asseler J, Milani AL, Vollebregt A, Roovers JP. Effect of pessary cleaning and optimal time interval for follow-up: a prospective cohort study. Int Urogynecol J. 2020;31(8):1567-74. https://doi.org/10.1007/s00192-019-042008.

14. Melon J. Commentary on "effect of pessary cleaning and optimal time interval for follow-up: a prospective cohort study.". Int Urogynecol J. 2020;31(8):1575. https://doi.org/10.1007/s00192019-04211-5.

15. Anderson KE, McGinty EE, Presskreischer R, Barry CL. Reports of forgone medical care among US adults during the initial phase of the COVID-19 pandemic. JAMA Netw Open. 2021;4(1): e2034882. https://doi.org/10.1001/jamanetworkopen.2020.34882.

Publisher's note Springer Nature remains neutral with regard to jurisdictional claims in published maps and institutional affiliations. 